\title{
A Massively Parallel Re-Configurable Mesh Computer Emulator: Design, Modeling and Realization
}

\author{
Mohamed YOUSSFI ${ }^{2}$, Omar BOUATTANE ${ }^{1}$, Mohamed O. BENSALAH ${ }^{2}$ \\ ${ }^{1}$ E. N. S. E. T, Bd Hassan II, BP, Mohammedia Morocco; ${ }^{2}$ Faculté Des Sciences Université Mohamed V Agdal Rabat Morocco. \\ Email: o.bouattane@yahoo.fr,med@youssfi.net
}

Received November $4^{\text {th }}, 2009$; revised November $26^{\text {th }}, 2009$; accepted December $19^{\text {th }}, 2009$.

\begin{abstract}
Emulating massively parallel computer architectures represents a very important tool for the parallel programmers. It allows them to implement and validate their algorithms. Due to the high cost of the massively parallel real machines, they remain unavailable and not popular in the parallel computing community. The goal of this paper is to present an elaborated emulator of a 2-D massively parallel re-configurable mesh computer of size $n \times n$ processing elements (PE). Basing on the object modeling method, we develop a hard kernel of a parallel virtual machine in which we translate all the physical properties of its different components. A parallel programming language and its compiler are also developed to edit, compile and run programs. The developed emulator is a multi platform system. It can be installed in any sequential computer whatever may be its operating system and its processing unit technology (CPU). The size $n \times n$ of this virtual re-configurable mesh is not limited; it depends just on the performance of the sequential machine supporting the emulator.
\end{abstract}

Keywords: Parallel processing, Object Modeling, Re-Configurable Mesh Computer, Emulation, XML, Parallel Virtual Machine

\section{Introduction}

Recently, in the data analysis and signal processing domain, the analysis tools, the computation methods and their technological computational models, have known a very high level of progress. This progress has oriented the scientists toward new computation strategies based on parallel approaches. Due to the large volume of data to be processed and to the large amount of computations needed to solve a given problem, the basic idea is to split tasks and data so that we can easily perform their corresponding algorithms concurrently on different physical computational units. Naturally, the use of the parallel approaches implies important data exchange between computational units. Subsequently, this generates new problems of data exchange and communications. To manage these communications, it is important to examine how the data in query are organized. This examination leads to several parallel algorithms and several corresponding computational architectures. Actually, we distinguish several computer architectures, starting from a single processor computer model, until the massively fine grained parallel machines having a large amount of processing elements interconnected according to several topological networks. Indeed, the analysis of the per- formance enhancement in terms of processing ability and execution speed must take into account the data computation difficulties and addressing management problem of these data. With relation to the last problem, it seems that the classical VON NEUMANN processor model is not able to respond to all the mentioned constraints. Furthermore, the optimized software realized for some cases have quickly demonstrated the limits of this model.

The need of the new architectures and the processor efficiency improvement has been excited and encouraged by the VLSI development. As a result, we have seen the new processor technologies (e.g. Reduced Instruction Set Computer "RISC", Transputer, Digital Signal Processor "DSP", Cellular automata etc.) and the parallel interconnection of fine grained networks (e.g. Linear, 2-D grid of processors, pyramidal architectures, cubic and hyper cubic connexion machines, etc.)

In this paper, our study is focused on a fine grained parallel architecture that has been largely studied in the literature and for which several parallel algorithms for scientific calculus were developed. From the theoretical point of view, each computational model has its motivations and its exciting proofs. In the practice, some models were technologically realized and served as real computational supports, but some others remain in their theo- 
retical proposition state. They are not realized because of their technological complexities and to their very high production cost. The computational model that is concerned in our work has known a very large technological progress. At first, it was viewed as a simple grid of cellular automata, after some technological enhancement, the cellular automaton became a fine grained processing element and the resulted grid became the mesh connected computer MCC [1]. Using some additional communication Buses, the MCC became the mesh with multiple broadcast [2] and polymorphic torus [3]. Finally, we speak about the reconfigurable mesh computer (RMC) that integrates a reconfiguration network at each processing element $[4,5]$.

From the algorithmic point of view, several authors were developed new parallel algorithms for data processing and scientific calculus. These algorithms were assigned to be implemented in the architectures mentioned above. Also, they are established using parallel approaches in order to reduce their complexities in term of execution times.

In order to facilitate for each parallel programmer the task of validation and program tests (even if he has not a real parallel machine that supports his algorithms), the emulating solutions were proposed in the literature to elaborate some virtual systems to perform the algorithms. The emulated systems may be specific as in $[21,22]$ or of general behaviors as in $[19,20]$.

In this context, we present in this paper a virtual tool to emulate an RMC, in which we can easily implement parallel SIMD algorithms. It is a virtual RMC platform of size $(\mathrm{n} \mathrm{m}$ ) processing elements (PE), where $\mathrm{n}$ is the number of rows in the matrix an $\mathrm{m}$ is the number of columns. Without loss of generality, we consider $n=m$ and we speak about a squared matrix of $\mathrm{n}^{2}$ PE's.

This emulator allows the scientists to overflow the problem of real RMC availability. (i.e. at this time the RMC machine is not yet popular due to its high cost).

Using this emulator, we propose an extended virtual RMC model, which translates all the functionalities of a real machine. This model can be easily extended to perform other advanced functionalities required by the multiplicity of the algorithmic techniques. In order to reach this virtual machine result, we started by the object modeling of all the components of the RMC such as the $n \times n$ grid, its processing element $\mathrm{PE}$, its connexion buses and so. To describe in more details the different steps of this emulator realization, we organize this paper as follows: Section II presents the real RMC model and the essential properties of its components. The object modeling of the RMC and some related details are given in section III. The next section, presents some parallel programs and their implementation on our virtual machine to illustrate the use of some established parallel instructions. Notice that, to avoid the upload of this paper, the complete set of instructions established for our platform is not presented in more details. We present just some examples of testing programs that involve some scientific computations such as basic matrix computation, data processing and image processing. Finally, the last section gives some concluding remarks and some exploiting perspectives of our virtual machine.

\section{Parallel Computational Model}

\subsection{Presentation}

The parallel architectures have known a large development these recent years. They are presented in numerous topological shapes, such as, linear, planar, pyramidal, cubic and hyper cubic networks. This large number of architectures requires an adequate classification taking into account several criteria. Among these criteria, we distinguish for example, the size of the machine, its autonomy of addressing and connexion, data type used etc. This classification allows the programmer to choose an appropriate computational model to perform the programs. Several proposed classifications were described in the literature; the diversity of the architectural solutions makes difficult the establishment of a general taxonomy. The well known classification is based on multiplicity of the instruction and data flows. It proposed four types of data machines, they are: the Single Instruction Single Data (S.I.S.D), single Instruction Multiple Data (S.I. M.D), multiple Instruction Single Data (M.I.S.D) and multiple Instruction Multiple Data (M.I.M.D) machines. Throughout this classification, the concerned model in this work is the Re-configurable Mesh Computer. It is the 2-D planar grid or matrix of $\mathrm{n} \times \mathrm{n}$ processing elements (PE). It is an S.I.M.D structure where in the word model the PE's use data buses of width at most $\log _{2} n$ bits. Also, in this model, the PE's has the autonomy of operation, addressing and connexion.

\subsection{Topology and Structure}

A Re-configurable Mesh Computer (RMC) of size $\mathrm{n} \times \mathrm{n}$, is a parallel machine having $\mathrm{n}^{2}$ Processing elements (PE's) arranged on a 2-D matrix as shown in Figure 1. It is a "Single Instruction Multiple Data (SIMD)" structure,

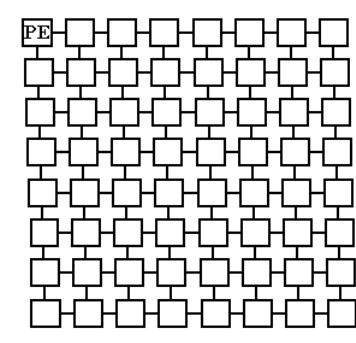

a) MCR "bit-model"

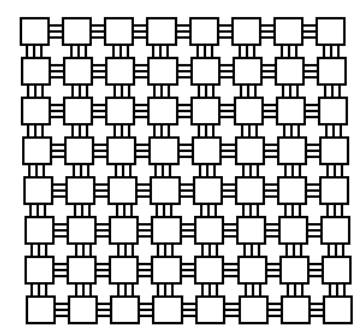

b) MCR "Word-model"
Figure 1. Re-configurable mesh computer model of size 8 × 8 
in which each $\operatorname{PE}(i, j)$ is localized in row $i$ and column $j$. It has an identifier defined by ID $=n x i+j$. Each PE of the mesh is connected to its four neighbors (if they exist) by communication channels. It has a finite number of registers of size $\left(\log _{2} n\right)$ bits. The PE's can carry out arithmetic and logical operations. They can also carry out reconfiguration operations to exchange data over the mesh.

The re-configurable networks that are presented as the processors matrix were improved considerably these last years. Indeed many theoretical and practical works appeared in the literature use this architecture as a computational model [6-8]. More particularly the recent related works propose new re-configurable models [9-14]. These re-configurable networks are based on a dynamic change of the mesh shape. They are qualified as the polymorphic grids of processors.

These architectures are provided with an instruction set of reconfiguration in order to get several topological shapes according to the problem to be solved. They are presented typically in the form of a multidimensional network of processing elements connected to a communication bus having a fixed number of wiring of Input/output. When this bus is reduced to only one bit width, we speak about "bit-model" machine, whereas for a mesh of size $n \times n$ having a bus of width $\left(\log _{2} n\right.$ bits), we speak about "Word-model" machine as in $[9,15]$. Figure 1, shows a 2-D representation of this model. Reconfiguration is locally made by adjusting the bus switches at each PE. The control of these switches offers to the PE's connection autonomy. Indeed, different PE's can simultaneously select various switches to achieve a given configuration. This is based on local decisions made by each PE. Also, it is possible for all the PE's of a selected group to carry out unconditional operations of configuration, where the PE's carry out reconfiguration instructions to activate their switches.

\subsection{Basic Operations of a PE}

\subsubsection{Arithmetic Operations}

Like any standard processor, the PE's of the RMC have an instruction set relating to the arithmetic and logical

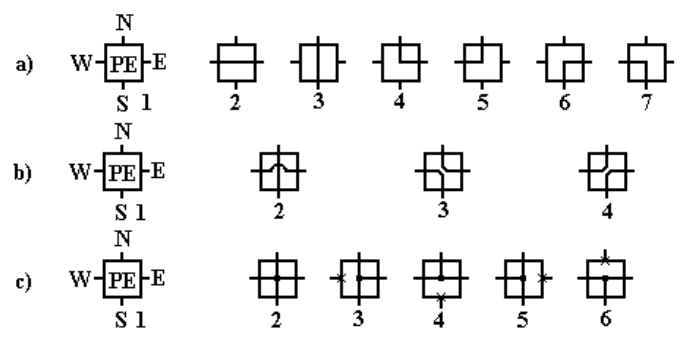

Figure 2. Different bridging configuration of a PE. a) Simple bridge, b) Double bridge, c) Crossed bridge operations. The concerned operands can be local data of a PE or the data received on its communication channels after an inter-PE data exchange operation. In the "bitmodel" machine of size $\mathrm{n} \times \mathrm{n}$, the calculation in the PE's is carried out bit by bit, whereas for the types "Wordmodel" of the same size, calculations are done on words of size at most ( $\mathrm{k}=\mathrm{L} \log \mathrm{B}_{2 \mathrm{~B}} n$ bits), where $\mathrm{k}$ is the width of the communication bus of the PE's.

\subsubsection{Configuration Operations}

In this part, we present the three kinds of bridging operations carried out by the PE's to facilitate the data exchange over the mesh. These configuring operation were largely exploited in several parallel algorithms to enhance the algorithmic complexities [16-18].

\subsubsection{Simple Bridge (SB)}

A PE of the RMC is considered in SB state when it establishes connections between two of its communication channels. This PE can connect itself to each one of its channel bits, either in transmitting mode, or in receiving mode. It can also isolate itself from some of its bits (i.e. neither transmitter, nor receiver). Various cases of SB figures are realized, they are: $\{\mathrm{EW}, \mathrm{S}, \mathrm{N}\},\{\mathrm{E}, \mathrm{W}, \mathrm{SN}\}$, $\{\mathrm{ES}, \mathrm{W}, \mathrm{N}\},\{\mathrm{NW}, \mathrm{S}, \mathrm{E}\},\{\mathrm{NE}, \mathrm{S}, \mathrm{W}\}$ and $\{\mathrm{WS}, \mathrm{E}, \mathrm{N}\}$.

E, W, N and S indicate the Port sides of a PE; they are: East, West, North and South respectively. Figure 2(a) shows the different configurations of the SB state.

\subsubsection{Double Bridge (DB):}

A PE is in a DB state when it carries out the configuration operations creating two independent buses by its communication channels. The different possible configurations that can be obtained are: $\{\mathrm{EW}, \mathrm{NS}\},\{\mathrm{ES}$, $N W\}$ and $\{E N, S W\}$. Figure 2(b) presents the different configurations of the DB state.

\subsubsection{Cross Bridge (CB):}

A PE puts itself in $\mathrm{CB}$ state by connecting all its active communication channels in only one bus. This operation is generally used when we want to transmit information to all the PE's of a connected component at the same time.

The $\mathrm{CB}$ state is defined by the unique configuration: \{NESW \}, where only one of the four ports of a PE can be locked, otherwise the $\mathrm{CB}$ state becomes an SB state, see Figure 2(c).

These various bridges are applicable on the two types of machines "bit-model" and "Word-model". Their establishments require the setting of the associated switching matrix at each PE.

\subsubsection{Inter Processors Operations}

The inter processor operations are principally classified in the data exchange category. Data exchange may occur between several pairs of PE's each others or between one or more PE's and a group of selected PE's. To illustrate 
the concept of inter processor operations, we present an example of data exchange procedure named "Direct broadcasting"

The "Direct broadcasting" procedure consists of transmitting information from a given PE over a mesh resulted by the CB operation, to all the connected PE's on this mesh. The complexity of this operation is: iteration. The necessary instructions are:

\section{All the PE's go to the CB state.}

- All the PE's couple themselves in receiving mode on the resulted bridge, except for the PE which will transmit data. It must be coupled in transmitting mode.

The transmitting PE transmits Data on its bridge. Thus all the receiver PE's can read concurrently the same information on their 'communication buses.

More details about the reconfiguration operations in technological point of view and communication cost are discussed in [3], where the polymorphic torus is equipped by the same reconfiguration network as the re-configurable mesh.

\section{Object Modeling of the RMC}

\subsection{Presentation}

As mentioned in the precedent section, the RMC is a set of processing elements arranged in a squared matrix. Each PE is modeled by an object defined by a state and a behavior.

The state of the PE object is represented by the values of its memory registers and its internal linked components, such as, its arithmetic and logical unit (ALU) and its four ports (East, West, North, South).

The PE behavior represents the set of operations that it can carry out. The kind of these operations is: arithmetic, logic, bus configuration (e.g. bridge operations) data exchange, marking and unmarking a PE, etc. In addition to these operations, it may be necessary to add other specific operations to delegate to a given PE a task to represent a given group of PE's in the RMC. In this case as in $[12,17]$, the delegated PE is labeled Representative PE (RPE).

Generally, all the basically operations required by a PE belong to its object behavior section. Taking into account the object modeling of all the PE features, it seems that this approach is the appropriate tool to realize our parallel programming emulator.

Since the emulator environment is performed on a sequential machine, a parallel to sequential mapping is needed. This means that the realized emulator requires three layouts:

The sequential layout: It is the kernel of our project. It performs all the operations of the emulating program.

The parallel layout: It represent the parallel programming RMC environment, where any parallel pro- grammer can write, compile, debug and run its parallel program.

The third layout is the intermediate one between parallel and sequential layouts. It realizes the mapping task, where each parallel instruction is converted into a set of sequential instructions.

Our parallel machine is represented by the instance of the platform class, which represents a mesh. This later is defined by a set of PE's arranged in an $\mathrm{n} x \mathrm{n}$ matrix. A $\mathrm{PE}$ represents an object of processor class. In order to communicate over the mesh, each processor object is linked to four objects of classes: EastPort, WestPort, Northport and Southport. These four classes have the same features; they are grouped in an abstract Port class using the heritage concept. Each of the four ports is linked by one communication bus to its neighboring PE ports. Each PE performs any computation using an arithmetic and logic unit. This unit is modeled by a structure defined in the $\boldsymbol{A L U}$ Unit class.

In the realized platform the parallel programmers must edit or open an edited parallel program and compile it before its execution. These later must be written using XML language according to an ad hoc developed XML scheme. The XML language is adopted to elaborate our system because it presents a powerful tool to model the complex components as the processing element. It was used in [23] to describe parallel grid components.

To construct a parallel program, some sets of instructions were elaborated with respect to the object programming rules, so that the whole program possesses a unique object representation. This representation translates the following concept scheme:

- $\quad$ A program is a set of instructions

- $\quad$ Each instruction is defined by a name, a set of attributes and can contain other instructions

Each attribute is defined by a name and a value.

The UML class diagram of Figure 3 shows the different classes of our principal model. Also, in this figure, we can see all the relations between the different object models of the real components of the RMC.

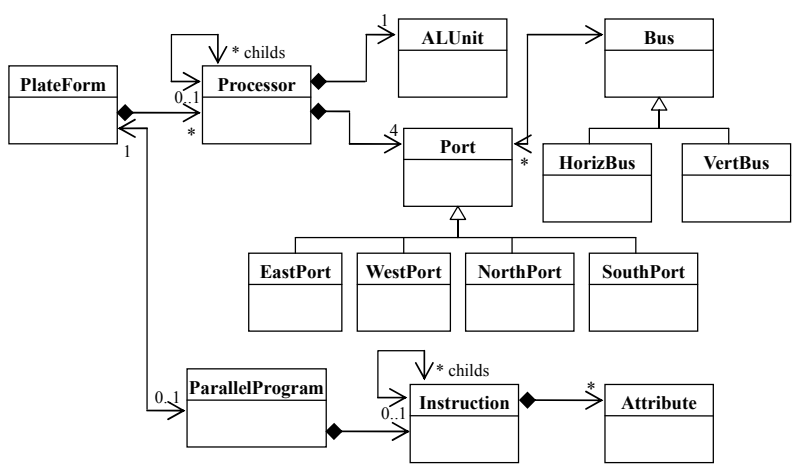

Figure 3. UML diagram of the modeling classes of the parallel platform 


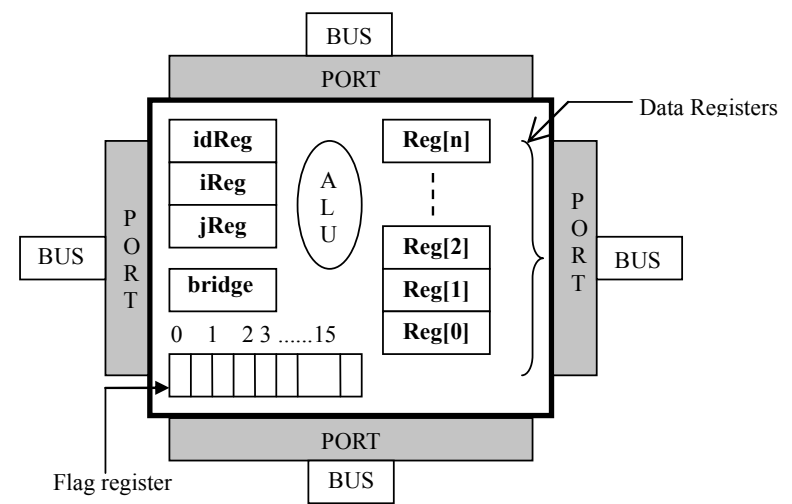

Figure 4. Representation of the components of a processing element model

\subsection{Description of the RMC Model Classes}

\subsubsection{Processor Class}

In this part, we have translated all the physical components of a PE as in Figure 4, and its functionalities to the processor model. The defined processor class must take into account all the elementary functions and the set of the elementary physical parts of the PE. Thus, each PE is defined by its state, its behavior and must have an identity.

\subsubsection{Processing Element State}

The state model of a PE describes all its physical components. They are:

\section{Identifier registers:}

When a created PE is inserted in the mesh model, its instance variables iReg and $j R e g$ which represent its location coordinates in the $\mathrm{n} \times \mathrm{n}$ matrix are set. The identifier register of this PE idReg, will take the value computer in the row major order by: $i d R e g=n * i R e g+j R e g$.

\section{Internal registers:}

For any given computation problem, each PE must use some internal registers to save data and the results of any related processing. To do so, we define in the PE model an array of internal data registers named "reg [..]". In this model, we have defined arbitrarily an array of 16 data registers. This array may be extended dynamically to any other size according to the problem in query.

\section{Flags:}

As any standard processor, we introduce in the PE model a special flag register, where each of its flag bits will indicate the PE state related to any performed instruction. This register is arbitrarily defined by an array of 16 bits, but it can be extended to any large size according to any additional useful instruction.

\section{Communication Ports:}

In the real RMC machine, all the PE's can exchange data via their communication ports, between each others what ever their locations in the matrix. In order to accomplish this task in the modeled machine, the commu- nication ports of a PE are represented by objects linked to the PE using the reference indicators stored in the defined variables: eastport, westport, northPort and southPort.

\section{ALUnit:}

To perform advanced logical and arithmetical operations, each PE uses its ALUnit. This later is modeled by an object of the class ALUnit and having a reference stored in the variable named ALUnit.

\section{Other components:}

Notice that the RMC machine emulator is an open system. It can be dynamically and easily extended in terms of register memories, communication bus width, and special functions according to the problem in query. For example: In the image processing domain and for some specific applications, some hierarchical strategies are often used to enhance the complexity of the corresponding algorithms. (e.g. q-tree based algorithms, component contour based algorithms, labeling etc.). In such cases, of special advanced programming applications, some algorithms require special additional variables or registers. The programmer has the ability to extend easily its computational model.

To illustrate the proposition of this part, we consider an example where a PE can be representative of a group of other PE's. To do so, we define in the processor class a collection of objects of type processor. In this collection we save the represented PE identifiers. Furthermore, a given PE can easily know its representative PE using the representative $\boldsymbol{P E}$ variable which contains the identifier of its representative PE. This representative property was largely used in the literature as in $[12,17]$.

\subsubsection{Processing Element Behavior}

The behavior of the PE is implemented by methods inside the processor class. These methods are classified in three categories.

Basically operations: The basically operations that can carry out a PE are the same as in any standard processor. Thus in this part we have implemented the methods to model the arithmetic operations (addition, subtraction, multiplication, division, etc.). Also we have implemented two operations to model some complex arithmetic and logic expressions. They are named: executeArithmeticExpression() and executeLogicExpression(). These expressions may respect the format imposed by $\mathrm{C}++$ and java programming language. To do so, we have created a logic and arithmetic expression compiler using a complex process. This process is not presented in this paper because it is not the main idea discussed in this work.

Several other methods have been defined in this class to offer to the programmer a large library of useful methods and operations.

Data exchange operations: The previous operations can be viewed as a category belonging to the set of instructions of any sequential programming language. Par- 
allel programming is characterized by other types of instructions. In fact, as mentioned above, the Peps of the RMC must collaborate between each others by exchanging data via their communication buses. Among the implemented methods of this category, we distinguish:

- $\quad$ sendValue (double v): This method allows the $\mathrm{PE}$ to send a data value $\mathrm{v}$ to its neighboring PE's according to its bridge configuration state.

- $\quad$ receiveData (double data, char port, byte regR): Allows the PE to receive a data value on its port specified by the parameter port and to store the received data in its register $\operatorname{reg} R$.

- $\quad$ sendAndReceiveData (char portS, byte regS, char portR, byte regR): allows the $\mathrm{PE}$ to send the data value of its register regS on the port specified by the parameter port $S$, and receive data on the port specified by the parameter port $R$, the PE stores the received data in its register regR.

- $\quad$ receiveAndSendWithOperation (ProcessPort po$r t$, String op): This method is used to receive data, execute some operations on this data and send the result to other PE's according to their bridge states.

Configuration operations: In the parallel programming domain and particularly in the SIMD computational models, the processing element can play several roles depending on its configurations. In the processor class, we have defined a set of methods to change and to know the actual configuration of the PE. During the execution phase of a given parallel program, it is necessary to point at each stage of this phase, the group of active PE's. For these reasons, we define the methods select() and unselect() to select or unselect the Peps that are susceptible to execute or not the instructions. Among the selected PE's, it is possible to assign for some specific instructions the concerned PE's using other methods to mark or unmark them. Thus, we define other methods, mark() and unmark() to distribute a Boolean label to each selected PE. This label can be integrated in the program as a test variable.
To determine the configuration state of a PE at any given stage, some other appropriate operations are introduced.

The methods associated to the configuration operations depend usually on the content of the flags register. Figure 5 summarizes the significance and the usefulness of each bit of this register where:

\subsubsection{ALUnit Class}

In a given parallel program, the PE's must be able to perform the arithmetical and logical expressions defined by any mathematical expression using all the possibilities of a programming language like java. During the compilation procedure, the mathematical expressions must be compiled and stored in temporary files to be used during their execution. The static methods defined in the ALUnit class named, createArithmeticExpression() and createLogicExpression() are used to compile the arithmetic and logic expression. To run the previous compiled expression, we can use the following two methods:

- $\quad$ getLogicExpression(int index,ArrayList params): returns the result of the logical expression of the instruction numbered by the index parameter. If this expression is included in an iterative loop, the iteration values will be stored in the array params.

- executeArithmericOperation(int index, ArrayList params): executes the arithmetic expression of the instruction numbered by index. If this expression is included in an iterative loop, the iteration values will be stored in the array params.

\subsubsection{Port Class}

As mentioned above, each PE possesses four communication ports. Each port is associated to a communication bus. In our model, we define four types of ports: East, West, North and South. All these four ports have the same characteristics; they are grouped in an abstracted Port class.

A port is an object having a state defined by a label $(\mathrm{N}, \mathrm{E}, \mathrm{W}, \mathrm{S})$ and a reference " process" to the processor object to which it belongs.

\begin{tabular}{|c|c|c|c|c|c|c|c|c|c|c|c|c|c|c|c|c|}
\hline Bit number & $\mathbf{0}$ & 1 & 2 & 3 & 4 & 5 & 6 & 7 & 8 & 9 & 10 & 11 & 12 & 13 & 14 & 15 \\
\hline State $(x=0$ or 1 & $\mathbf{x}$ & $\mathbf{X}$ & $\mathbf{x}$ & $\mathbf{x}$ & $\mathbf{x}$ & & & & & & & & $\mathbf{x}$ & $\mathbf{x}$ & $\mathbf{x}$ & $\mathbf{X}$ \\
\hline : & $\begin{array}{l}\text { Bit 0 } \\
\text { Bit 1 } \\
\text { Bit 2 } \\
\text { Bit 3 } \\
\text { Bit 4 } \\
\text { Bit 12 } \\
\text { Bit 13 } \\
\text { Bit 14 } \\
\text { Bit 15 } \\
\text { Bits 5 }\end{array}$ & $\begin{array}{l}: \text { in } \\
: \text { in } \\
: \text { in } \\
: \text { in } \\
: \text { in } \\
: \text { in } \\
: \text { in } \\
: \text { in } \\
: \text { sh } \\
1 \text { ar }\end{array}$ & ates & $\begin{array}{l}\text { the } \\
\text { the } \\
\text { the } \\
\text { the } \\
\text { the }\end{array}$ & $\begin{array}{l}\text { te o } \\
\text { has }\end{array}$ & $\begin{array}{l}\text { ee } P \\
\text { ade } \\
\text { rep }\end{array}$ & $\begin{array}{l}\text { is } \mathrm{cl} \\
\text { lata }\end{array}$ & $\begin{array}{l}\text { ged } \\
\text { not. }\end{array}$ & not & . & not. & & & & & \\
\hline
\end{tabular}

Figure 5. The content of the flags register of a modeled PE 


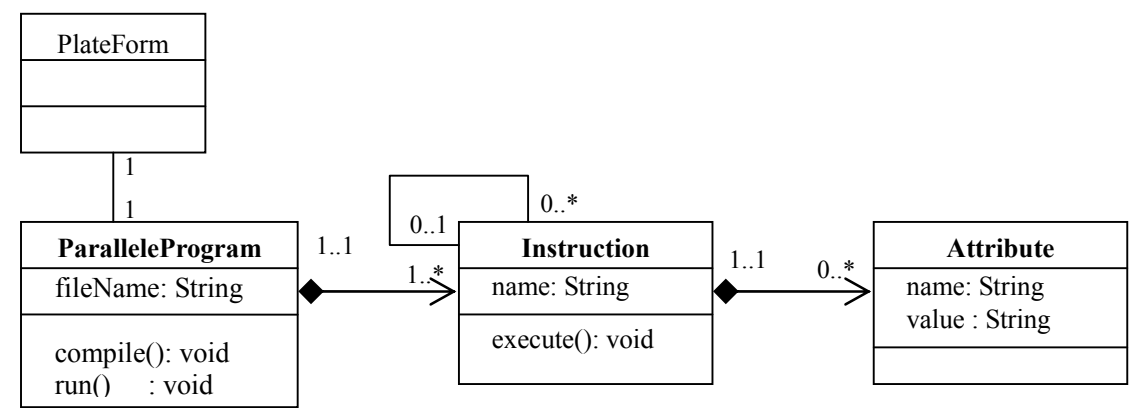

Figure 6. The UML class diagram of a program

A port can be locked by its PE using a Boolean instance variable locked. Since each port is associated to a communication bus, the reference to this bus must be stored in this class. Finally, each port must have one configuration variable to specify how the data can be transferred from the port to its communication bus.

Example: If we consider an 8 bits communication bus, the defaults configuration of a port is defined by the order $« 01234567 »$. This means that the bit $i(i=0$ to 7$)$ of the port is linked to the bit $\mathrm{i}$ of the bus. In this case the data sent over the port is transmitted without any transformation. While in the configuration «70123456» the bit 0 of the port is connected to bit 1 of the bus and the bit $i$ ( $i=1$ to 6 ) is linked to bit $i+1$ of the bus. The bit 7 of the port is linked to bit 0 of the bus. This means that the transmitted data is left rotated over the bus. This kind of commutation operations on ports are often used in several parallel programs [9].

\subsection{Parallel Program Object Modeling}

A parallel program loaded in the plate- form is composed by a set of instruction. Each instruction is defined by zero or several attributes. An instruction contains the child instructions and belongs to a father instruction. The class sheet of Figure 6 shows the class diagram of this model.

\subsubsection{Parallel Program Class}

A parallel program is defined by the source file name of this program. This source file is created by the programmer with the extension ".par" and must respect the XML norm. The program is defined by the variables: firstInstruction, lastInstruction and currentInstruction that represent the first, the last and the current instruction of the program (respectively). Some other instance variables are defined like: endCompilation to indicate if the compilation of the program is finished. iterationLevel to store the current iteration level in the loop cases.

To run an edited program, we begin by the method compile(). The compiling procedure acts as follows:

- $\quad$ read the program source file

- $\quad$ load it in the memory as a tree of instructions

- $\quad$ The first instruction is stored in the firstinstruction variable.
- $\quad$ The last instruction is stored in the lastInstruction variable.

- $\quad$ Execute the program starting from the first instruction.

\subsubsection{Instruction Class}

To edit a parallel program in this emulator, we have created a parallel programming language using our specific XML scheme. Each instruction of this language is defined by several attributes and can contain other instructions. Each attribute is an object of the attribute class which is self defined by its name and its value. In the program, each instruction is viewed as a tree structure. It is delimited by a beginning and ending tags. Between these tags, we can find other child instructions having the same structure. Between the tags of the child instruction, we can find other child instructions having the same structure etc. For this reason, an instruction is defined by a dynamic table named childs, to store the references to the other child instruction objects. Furthermore, an instruction can know its father thanks to the variable "parent" which contains its parent reference. Finally, there are other interesting variables used during the execution of the instruction, they are defined in this class, such as, opened and executed variables that indicate if the instruction is opened or executed.

\section{Example:}

$$
\begin{aligned}
& <\text { for-eachPE test }=\text { "reg }[0]>0 "> \\
& \quad<\text { mark }>> \\
& \quad<\text { doOperation expression="reg }[0]=(\operatorname{reg}[1]+\operatorname{reg}[2]) / 2 " /> \\
& \quad<\text { for-eachPE }>
\end{aligned}
$$

In this example scheme, we show the structure of the parallel instruction «for-eachPE». It is defined by an attribute «test» having the value «reg[0]>0». This instruction contains two instructions: The first is «mark» which is without attribute, the second is the «doOperation» instruction having an attribute of name «expression» and value $\langle\operatorname{reg}[0]=(\operatorname{reg}[1]+\operatorname{reg}[2]) / 2 »$.

This simple program example selects in the matrix of processors all the PE's having a positive value in their registers reg[0]. The selected PE's are marked (labeled). 
Then, all these marked PE's perform the arithmetic operation on their own data registers, reg[1] and reg[2]. Finally the result of each $\mathrm{PE}$ is stored in its reg[0] register. Figure 7 presents the object structure of the precedent example after its compilation.

\subsection{Platform Class}

The modeled parallel machine is represented by an object of the Platform class. In this paper, we are interested in the implementation of the mesh as a matrix of $n \times n$ PE's. But, we can easily extend the model to other architectures such as: Pipe line, Pyramidal machine etc. The RMC model is characterized by the rows and cols variables that define the size of the matrix. The matrix creation is made by a matrix process[rows][cols] of object processor $(\mathrm{PE})$. The size of the resulted virtual mesh is dynamically configurable and is not limited. It depends only on the available memory space in the used sequential machine supporting the emulator.

In addition to the PE's matrix, the platform is associated to an object representing the loaded and compiled parallel program. Some additional Boolean variables are used to complete the Platform state such as:

Debug, to indicate whether the step by step execution mode is activated

- DrawMode, to indicate the type of the graphic context used in the displaying data zone.

- Compile, to indicate whether the loaded program is compiled or not.

\section{- Etc.}

The most important part of this emulator is implemented in the behavior part of the class platform. After loading a program using the method loadProgramme(), we can run the compile() method to construct the instructions tree of the parallelProgram Object. For each of these constructed parallel instructions trees, we realize the parallel to sequential mapping task. Subsequently, this task translates each parallel instruction into a set of iterative sequential instructions.

In order to exploit the platform class to run a given parallel program, we classify all its defined methods into the following four categories:

\subsubsection{Loading data on the mesh}

In this category, we use some methods to load data in the mesh which is the matrix of PE's before any processing procedure. As example, we distinguish the following:

- loadImage(): loads data image of any format (bmp, jpg, png, gif) in the matrix of PE's.

- $\quad$ loadMatrix(): loads a data matrix from a text file to the matrix of PE's.

- loadRandomMatrix(): computes and loads a random data matrix in the mesh.

Notice that, all the used data types in any programming language are supported by the modeled PE's (e.g. Byte, int, long, float, double etc.).

\subsubsection{Configuration of the Mesh}

In the SIMD parallel programming domain, it is necessary to select the PE's that are susceptible to perform some given instructions. For this reasons, we have defined the selection methods basing on different criteria. We will discuss some ones in the instruction set part. After any selection, the method executeConfig(String conf) asks the selected PE's to execute a configuration operation.

\subsubsection{Execution of the Program}

After loading data and configuring the mesh, we can perform the other instructions of the parallel program related to the loaded data. The principal method used in this part is ExecuteInstruction (Instruction ins). This category of instructions concerns all the data processing and scientific calculus operations.

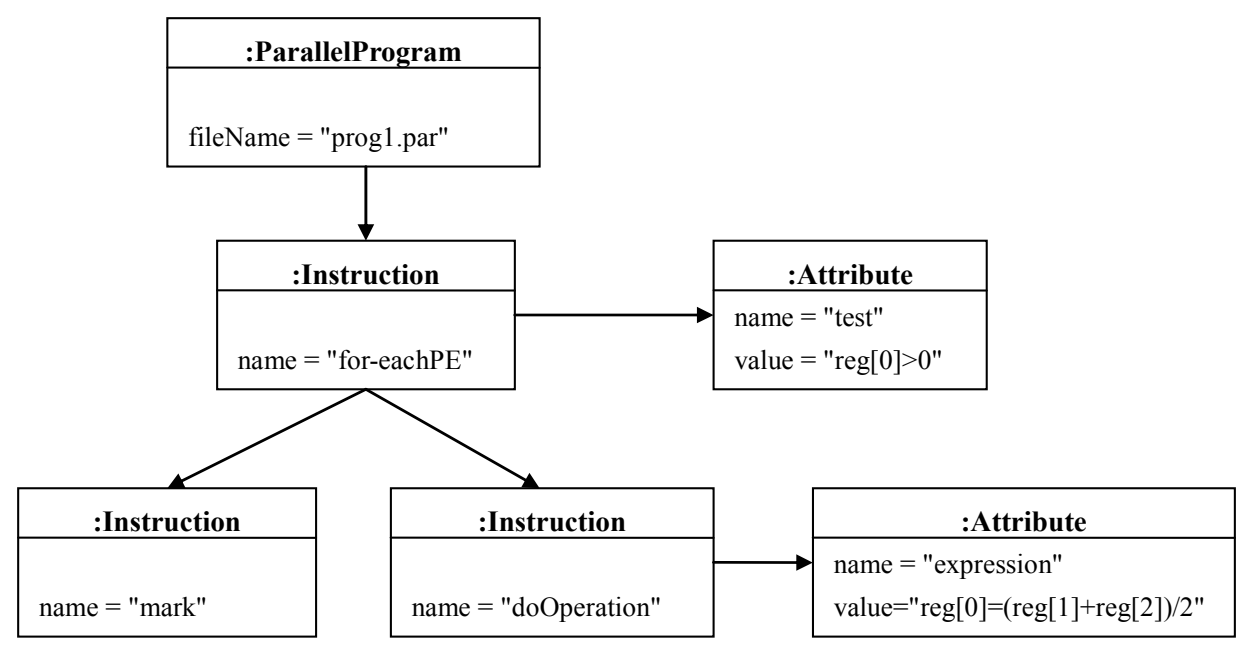

Figure 7. Object representation of an instruction 


\subsubsection{Displaying Results}

During the program execution, we can insert some methods to display the results using several numerical formats. For example, we can use the text format to observe the contents of different registers of the PE's or to display any data results in text format. A graphical context is also available in our platform to display other results in graphical mode such as curves and images.

\subsection{Instruction Sets}

In order to construct our programming language, we have developed an XML scheme which defines the parallel instruction sets to edit parallel programs.

A program must begin by the tag $<$ prog $>$ and ends by $</$ prog $>$ tag. Between the two tags we can edit the instructions.

Each instruction begins by the tag indicating the instruction name and ends by the tag indicating the end of the instruction. An instruction can be defined by several attributes, it can encapsulate other instructions.

As for the real RMC, the emulating platform has three categories of instruction sets. They are presented as follows:

\subsubsection{Instructions for PE Configuration}

In the SIMD architectures, the parallel programming is based on a fundamental principle, where it is necessary to select, mark and bridge the PE's concerned by the instruction execution. The configuration instruction set is expressed by the following actions:

Selecting: This action is expressed by the instruction:

$<$ for-eachPE cols $=$ "valCols" rows = "valRows" test $=$ "expression_logic" direction="'>>

\section{$</$ for-eachPE $>$ \\ ... (Operations to be executed)}

The attributes "cols" and "rows" define the concerned columns and rows. When several columns and rows are concerned, they are separated by commas.

The attribute direction is defined by a string to indicate the direction of the selected group of PE's starting from the PE of coordinates indicated in "rows" and "cols" attributes. The possible direction attributes are:

- $\langle\mathbf{R E} »$ or $« \mathbf{R W} »$ : to define the direction East or West along a Row.

- $\quad\langle\mathbf{C N} »$ or $« \mathbf{C S} »$ : to define the direction North or South along a Column.

- «DNE» or «DNW»: for the direction North-Est or North-West along a diagonal.

- «DSE» or «DSW»: for the direction South-Est or South-West along a diagonal.

The attribute test is used to select a set of PE's satisfying the condition defined by a logical expression.

For example, in the expression (test="reg[1]>10"), the selected PE's are those having in their reg[1] the values greater than 10 .

Marking: This action uses the following instructions:

$<$ mark type="true $\mid$ false" $/>$ : to mark a PE if its attribute type is true and unmark it, if this attribute is false.

$<$ mark $>$ : to mark a PE without condition.

$<$ unMark $>$ : to Unmark a PE without condition.

Bridging: As mentioned in the physical description of the RMC, a PE can be configured in three kinds of bridges: simple, double or crossed bridge. These bridging states are implemented by the following instruction:

$<$ bridge type $=$ "SB-WE|SB-NS $\mid$ SB-WN $\mid$ SB-WS | SB-NE | SB-SE | DB-WN-SE | DB-NE-SW |DB-NS-WE | CB-WNE | CB-NES | CB-ESW | CB-SWN | CB-WNES | $\mathrm{NB} " />$.

This instruction named bridge is used by a PE by defining its kind in the attribute type.

The attribute type is formulated by:

- Defining the kind by: $\mathrm{SB}=$ Simple Bridge, $\mathrm{DB}=$ Double Bridge and $\mathrm{CB}=$ Crossed Bridge.

Defining the direction of the bridge E,W,N and

$\mathrm{S}$ for East, West, North and South (respectively)

The SB configurations are: $\{$ SB-NS, SB-WE, SB-WN, SB-WS, SB-NE, SB-SE, SB-WS $\}$.

At any mentioned configuration the data is bypassing bi-directionally the PE over its bridges.

The DB configurations are: \{DB-WN-SE, DB-NESW, DB-NS-WE\}.

In this case, two communication bridges are constructed by a PE. So, two data can simultaneously bypass bi-directionally the PE.

The CB configurations are: $\{$ CB-WNE, CB-NES, CBESW, CB-SWN, CB-WNES\}.

In this case, the only one obtained communication channel links at least three ports of the PE.

Notice that, to return back to the no bridge state from any bridge configuration, we use the instruction bridge with an attribute type $\{\mathrm{NB}\}$ to specify No Bridge.

\subsubsection{Arithmetic / Logic Instructions and Control Structures}

In the modeled parallel programming language, the arithmetic and logic instructions are expressed using the format defined bellow. In addition to the reconfiguration operations, each PE of the RMC can carry out a set of arithmetic/logic instructions and control structures. These instructions are formatted as defined in the following examples:

\section{- Arithmetic/logic instructions}

$$
\begin{array}{cc}
\quad<\text { inc reg="numReg"/>: } & \text { means: reg [numReg } \\
=\operatorname{reg}[\text { numReg]+1; } & \\
\quad<\mathrm{dec} \text { reg="numReg"/>: } & \text { means: reg [num- } \\
\operatorname{Reg}]= & \text { reg [numReg]-1; }
\end{array}
$$


$<$ add reg="numReg" value= "val"/>: means : reg [numReg] = reg [numReg]+Value; $<$ sub reg="numReg" value= "val"/>: means : reg [numReg] = reg [numReg]-Value;

$<$ mult reg="numReg" value= "val"/>: means : reg [numReg] = reg [numReg]*Value; $<$ div reg="numReg" value= "val"/>: means : reg $[$ numReg] $=$ reg $[$ numReg $] /$ Value;

$<$ doOperation expression $=$ "arithmetic_expression" $/>$ : This instruction executes the arithmetic expression specified by the expression attribute. We have developped a compiler to execute all the possible arithmetical expressions according to the same syntaxes of the $\mathrm{C}$ and Java language.

\section{- Control structures}

The control structures defined for our platform are the same as the well known for any language. The following examples give the presentation of some structures.

$<$ if test= "logic_expression" $>\quad \ldots$ instructions ... $<$ if $>$

$<$ for from $=$ "begin_val" to $=$ "end_val" $>\ldots$ instructions ...</for $>$

$<$ while test $=$ "logic_expression" $>\ldots$ instructions ...</while $>$

\subsubsection{Data Exchange Instructions}

The third instruction set developed for this emulator concerns the specific instructions to exchange data between the PE's of the RMC. This set allows the programmer to manipulate some new concepts introduced in the parallel SIMD algorithms. These instructions use the four Port objects of each PE to send, receive and broadcast data over the mesh. Among the defined instructions we have:

$<$ sendAndReceiveData portS="W|E $|\mathrm{N}| \mathrm{S} " \quad$ regS="" portR="W|E|N|S" regR=""'/>

This instruction allows the PE to send it Data from the specified register regS to the port specified by portS and to receive data from protR and save it in the register regR.

$$
\begin{aligned}
& \quad \text { receiveAndTransmitData portS="W|E }|\mathrm{N}| \mathrm{S} " \quad \operatorname{regR} \\
& =\text { " " data=" " } />
\end{aligned}
$$

This instruction indicates to the PE to receive data specified by data attribute and store it in register regR then send it from the port specified by prots.

\subsection{Macro Commands and Parallel Functions}

As any programming language, the macro commands and functions are generated and inserted in specific libraries to facilitate their use in some advanced procedures. In the same strategy, we have defined some parallel macro commands and some functions to represent some parallel pre-processing procedures which can be inserted easily in any parallel program. The generated library is subject to more extensions and developments. In this section, we present some examples of Macro commands and parallel functions to illustrate their usefulness as the pre-processing procedures.

\section{Macro commands:}

- <defineRepresentativePE-forEachRow />

This macro command is used to determine a representative PE of a group of marked PE's for each row of the mesh. This command it based on the Minimum value search procedure at each row of the mesh. The output of this command is the minimal identifier value (idReg) found in at each group of marked PE's in each row of the mesh.

- $\quad<$ defineRepresentativePE-ForEachCol />

It is the same procedure used as in the precedent macro command. But the Minimum value search procedure is applied on columns instead of rows.

\section{- $\quad$ <initialiseRepresentivePE />}

This macro command is used to reset all the representative PE's. In this case there is no representative of any group of PE's in the mesh. This means that each PE is self representative.

- $\quad<$ doDistributeParityIndex from="W|E|N|S" $>$

This macro is used to distribute a special index named "parity index", alternatively to the marked PE's a group. It is an important macro command used as a pre-processing for some high level algorithms. This macro corresponds to a procedure based on bridge and reconfiguration operations where each marked PE receives on its specified port a Boolean label "0" or "1", and then it inverts it before sending it to its neighboring marked PE according to the bridge state in which it is set.

\section{Functions:}

- isSelected ( ): indicates if the PE is selected or not. It uses the bit 1 of the flags register.

- isChanged (): indicates if the state of the PE is changed or not. This method is very useful in some graphic refreshment procedures. It uses the bit 2 of the flags register.

- $\quad$ isTestValide( ): indicates if the last executed logical operation is valid or not.

- $\quad$ getParity (): shows if the parity flag of the PE is true or false. It uses the bit 15 of the flags register.

- isRepresentative( ): indicates if the PE is a representative or not. It uses the bit 14 of the flags register.

- hasReceivedData( ): indicates if the PE has received data or not. It uses the bit 13 of the flags register.

- isMarked( ): indicates if the PE is marked or not. It uses the bit 0 of the flags register.

- hasLoadedData( ): indicates if the PE has 
loaded data or not. It uses the bit 3 of the flags register.

- hasfinished( ): returns true value if all the PE's are in stand by mode. This function is called in the stopping test of some iterative loops.

- hasRepresentant( ):indicates if the PE has a representative PE or not.

- REGRep[numReg]: indicates the value of the numReg register of its representative.

\section{Applications}

In this section, we present some parallel algorithm examples where some properties of the RMC are used to examine how they are expressed using some instructions of the instruction sets defined above.

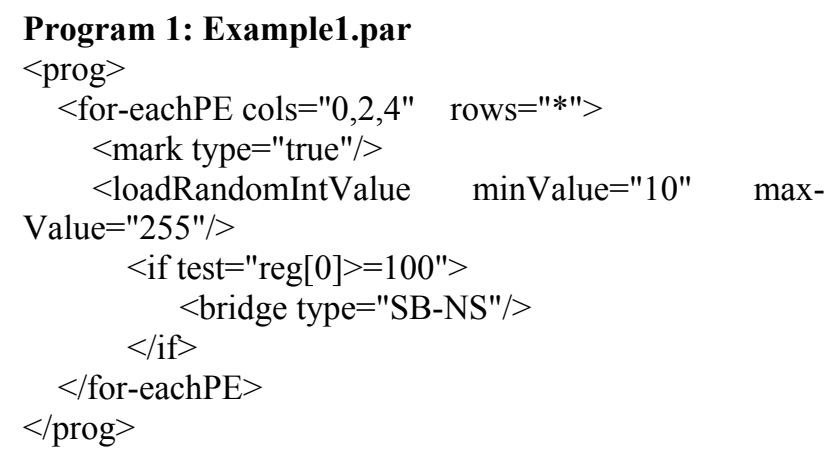

The result if this program is described as follows:

It selects all the PE's of the column 0, 2 and 4. Then all the selected PE's are marked "true".

Each one of these marked PE's must compute and save in its memory register reg[0], a random data having a value between minValue $=" 10 "$ and max-
Value $=" 255^{\prime \prime}$.

All the selected PE's having a data value greater than or equal to 100 must configure themselves in Simple Bridge of direction (North-South) NS.

Figure 8 shows the states of all the PE's of the RMC matrix after the program execution. The marked PE's are represented by the hashed squares. The NS bridge is represented by vertical lines linking its North and South ports of the PE is SB state and its neighbors.

\section{Program 2: Example2.par}

This example shows the point to point mean computation of two data matrices.

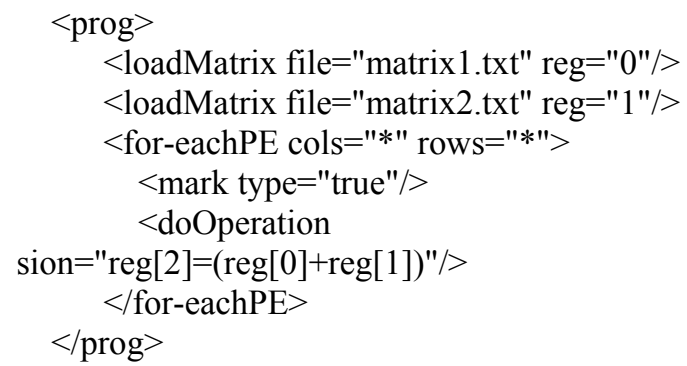

We consider two data matrices saved in the data files «matrix1.txt» and «matrix2.txt» respectively. The results of the different stages of this program example are:

The first matrix is mapped to the RMC of the same size as this matrix, one element per PE. At each PE the element is stored in its Reg [0] register.

- $\quad$ The second matrix is mapped to the RMC of the same size as this matrix, one element per PE. At each PE the element is stored in its Reg [1] register.

- $\quad$ All the PE's of the RMC are selected and marked.

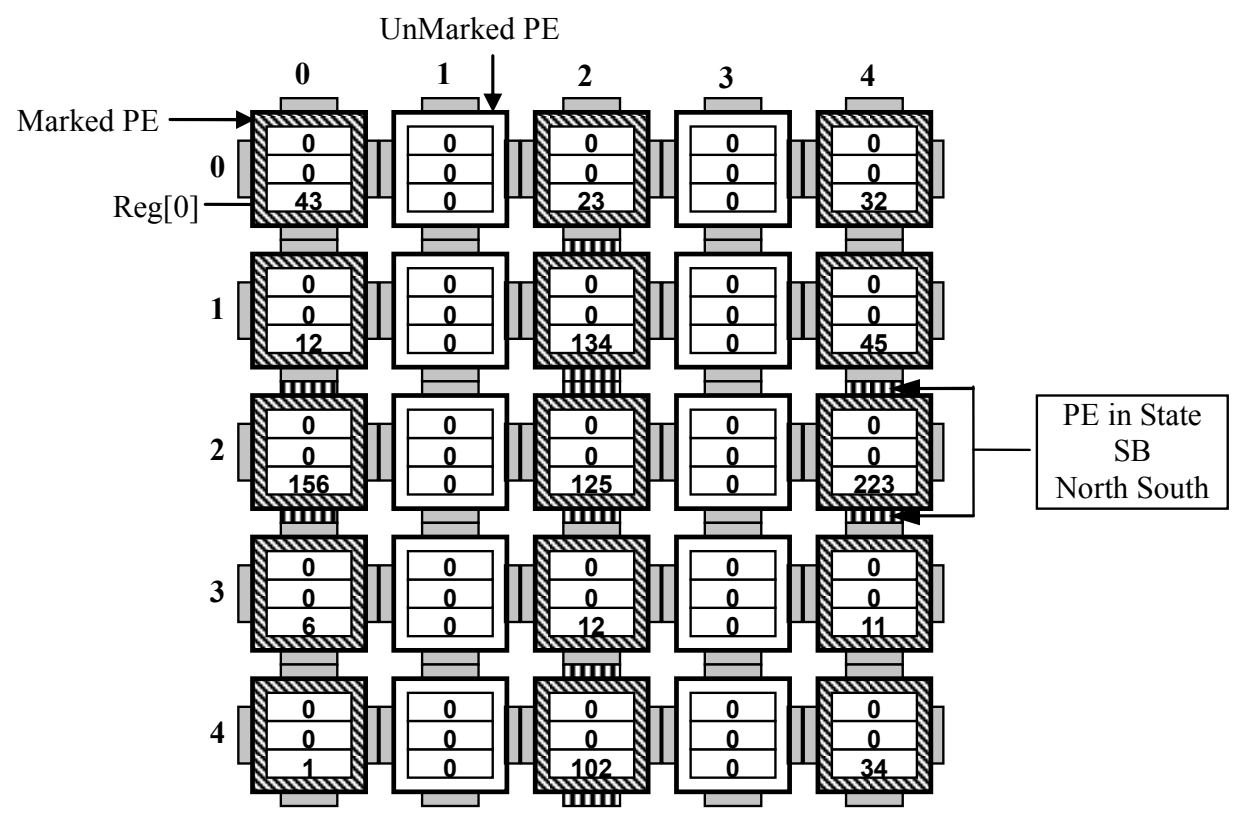

Figure 8. The different PE's states after program execution 


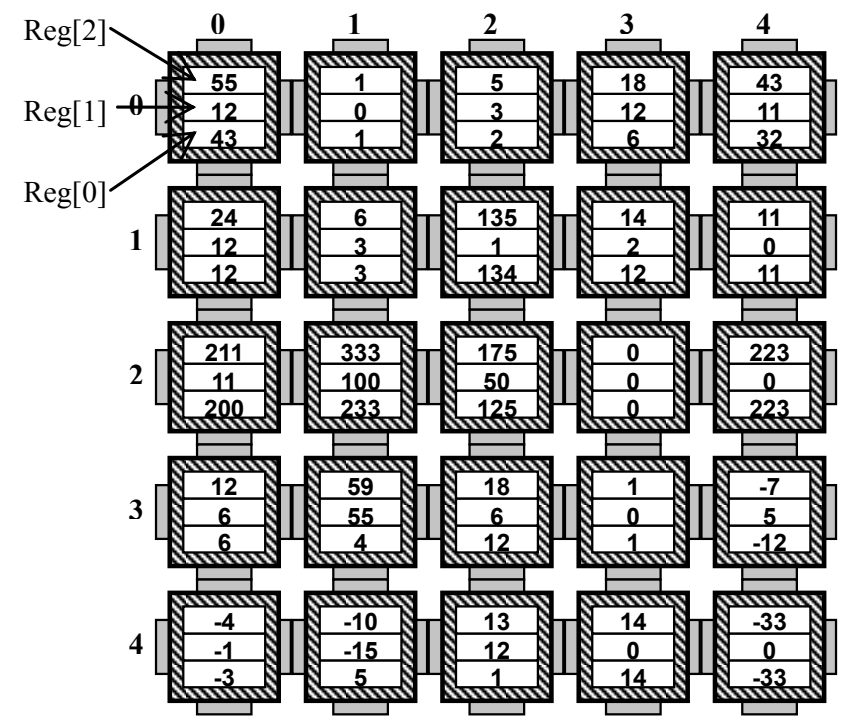

Figure 9. Result of the point to point sum of two matrices on the modeled RMC

All the marked PE's execute simultaneously the operation $(r e g[2]=r e g[0]+r e g[1])$. This is the point to point mean computation. At each $\mathrm{PE}$ the result is saved in its reg[0] register.(Figure 9)

\section{Program 3: Example 3.par}

This example corresponds to an implementation of a component contour detection of a gray leveled image using Sobel operator. The used image is of size $200 \mathrm{x}$ 200 pixels.

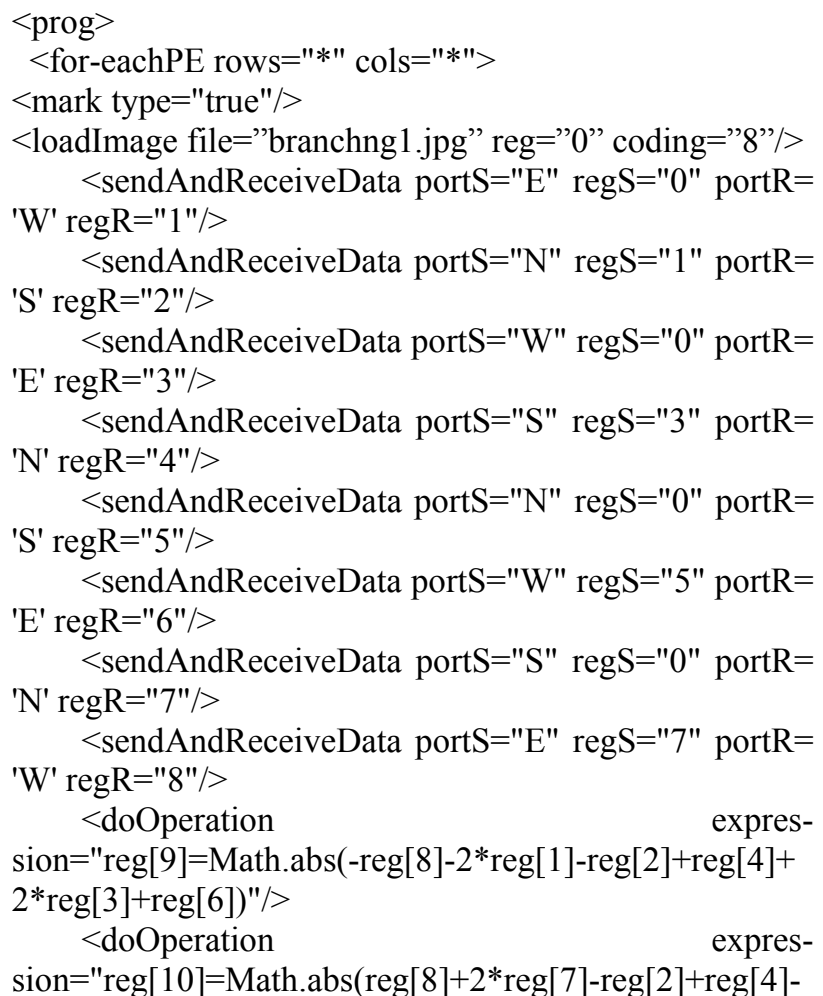

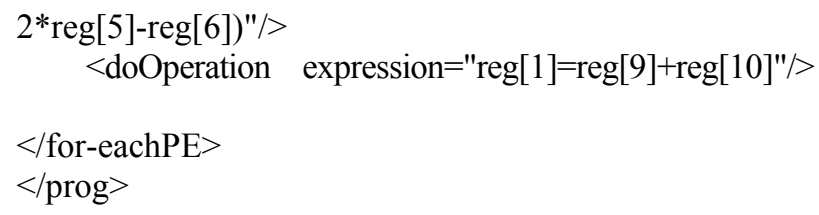

The execution of this program is commented as follows: 200) RMC.

Selecting and marking all the PE's of the $(200 \mathrm{x}$

The image is loaded from file="'branchng1. jpg" and stored in the RMC one pixel per PE. Each PE saves its own gray level pixel (coding="8”) in its register $\operatorname{reg}[0]$.

The following eight "sendAndReceiveData" instructions are used by the PE's to exchange data between neighboring PE's, so that each $\mathrm{PE}$ will receive its eight neighboring pixel values required by the Sobel operator.

After the data exchange stage, we compute the absolute values of Gx and Gy of the Sobel operator

- The final result is computed by: expression= "reg[1]=reg[9]+reg[10]"

- The resulted image is located in the RMC, one pixel per PE (i.e. in reg[1] of each PE.), while the input image is still in reg[0].

Figure 10 shows the result of the parallel program of a component contour detection of a gray leveled image using Sobel operator. Each used images is of size $200 \mathrm{x}$ 200 pixels. The images in Figures 10(a), (b) and (c) representing flower, fishes and a tree branch (respectively) are the input images of the program. The resulted output images are shown in Figures 10(d), (e) and (f).

Program 4: Example 4.par

Parallel program of the hierarchical Minimum search algorithm: 


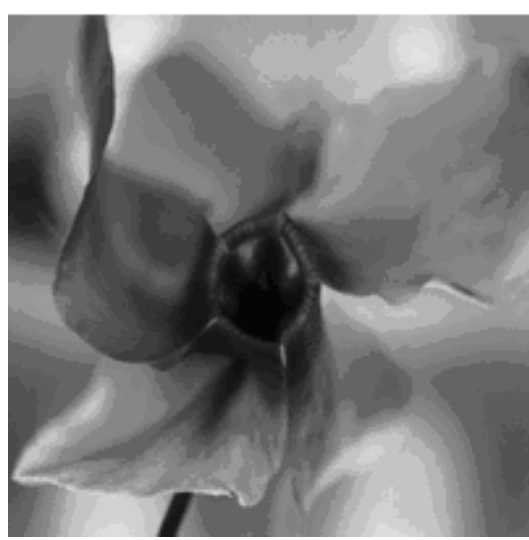

(a)

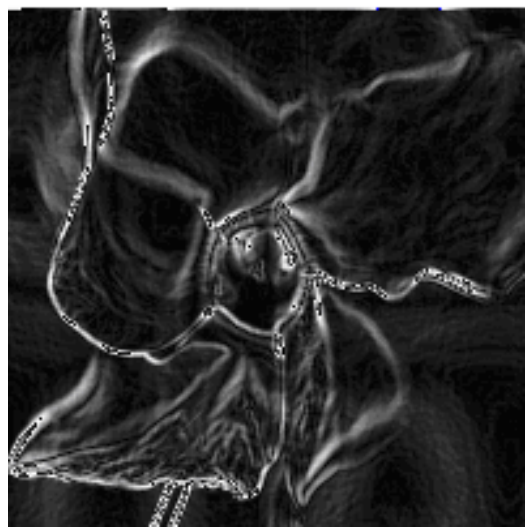

(d)

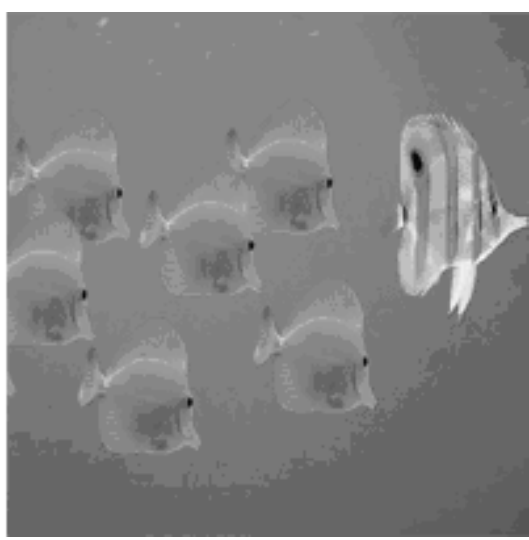

(b)

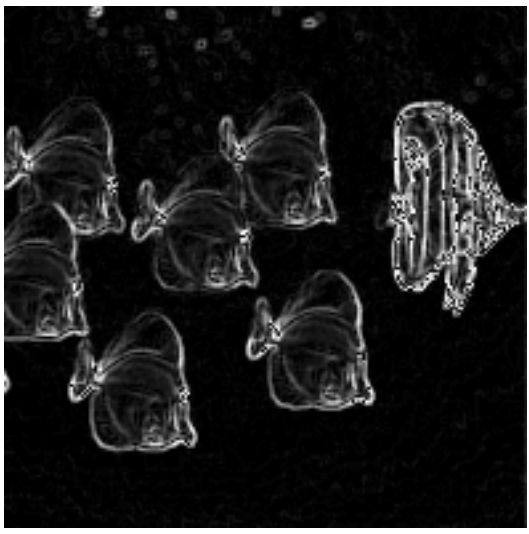

(e)

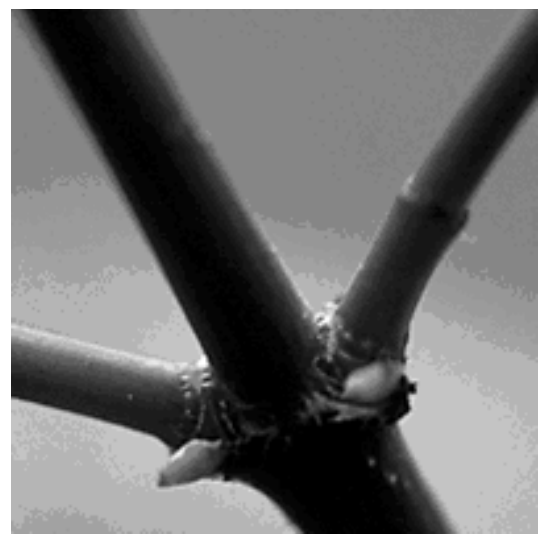

(c)

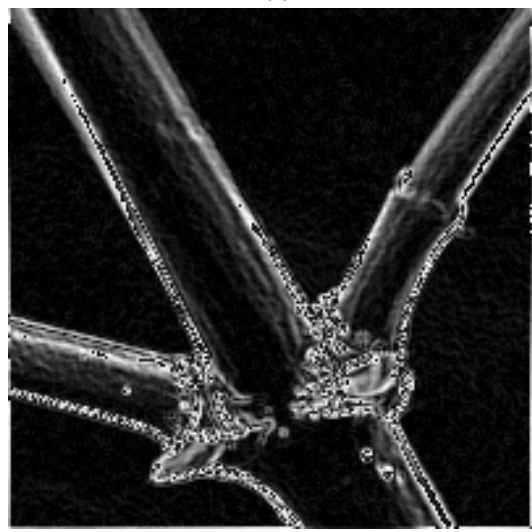

(f)

Figure 10. Results of the parallel program of contour detection using Sobel operator. The input images of the program are: (a) flower, (b) fishes and (c) tree branch, figures (d), (e) and (f) are the resulted output images (respectively)

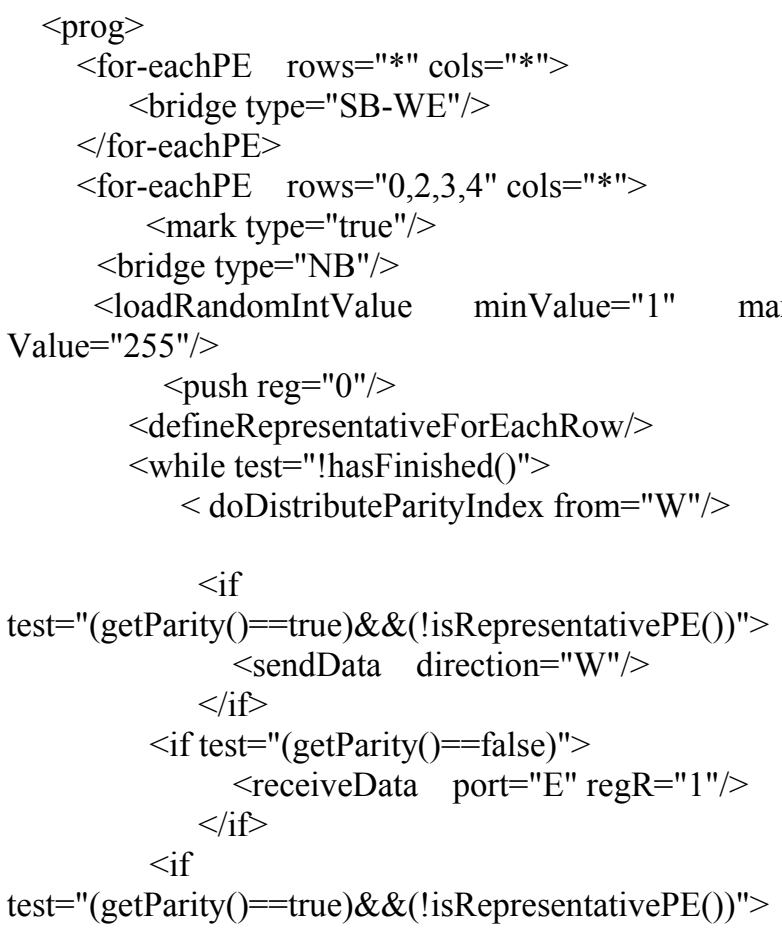

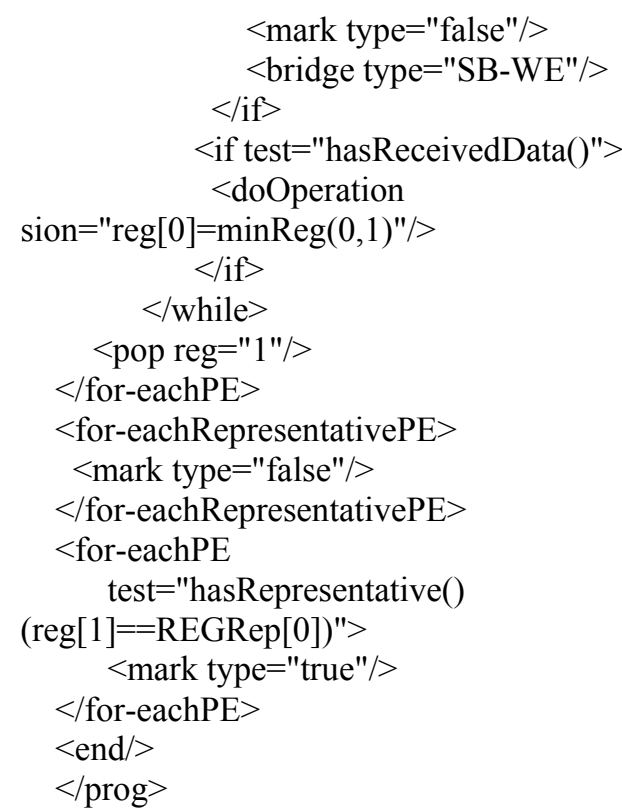

expres-

and

In this example, we use some sample instructions and control structures defined in the elaborated instruction sets. 


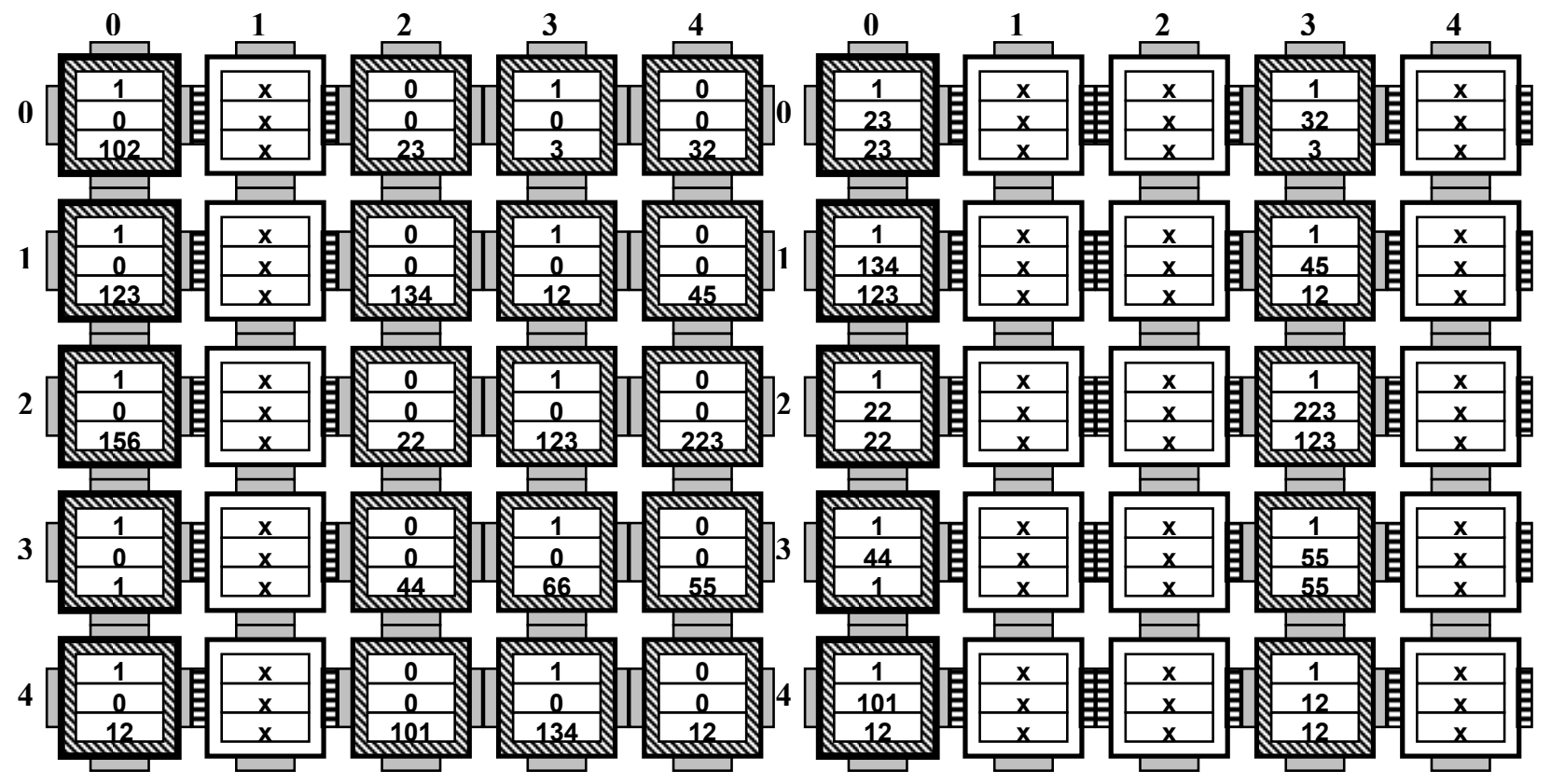

(a)

(b)

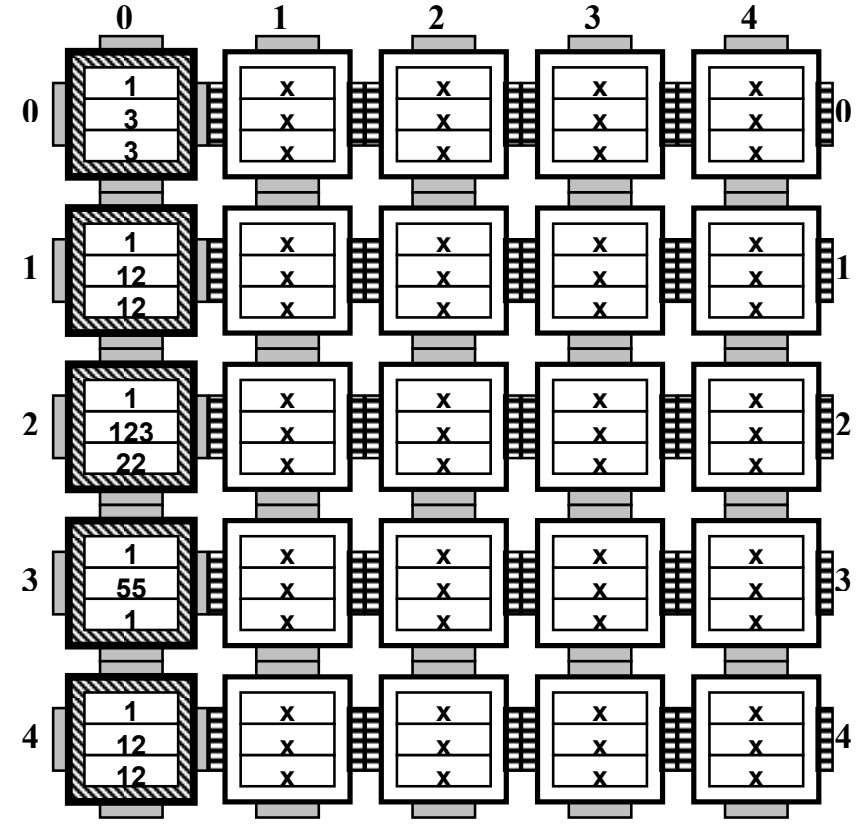

(c)

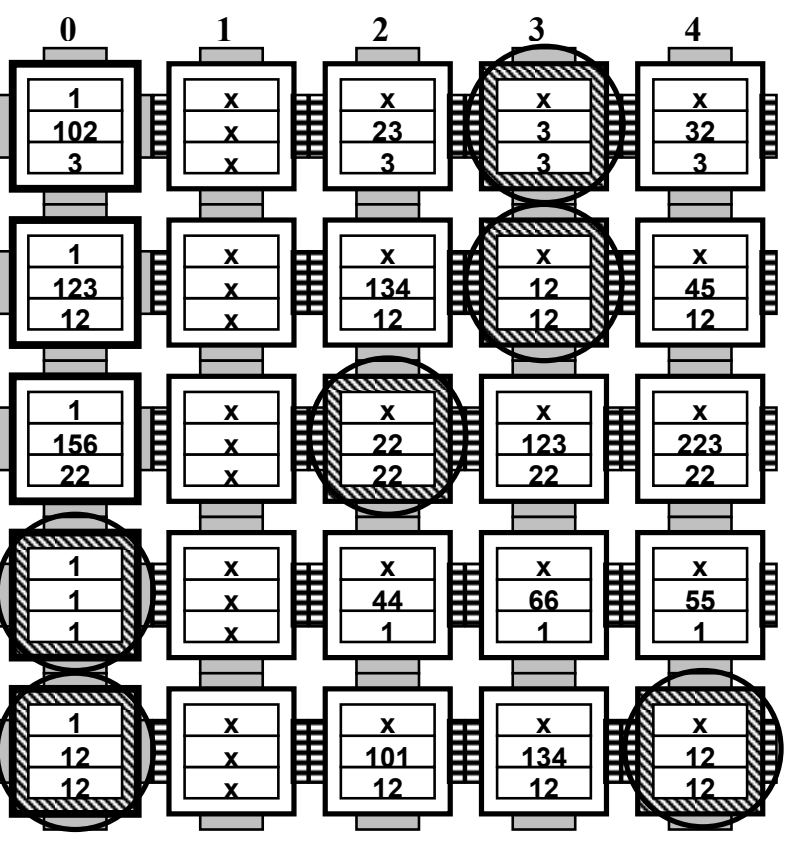

(d)

Figure 11. Result of a parallel minimum value search algorithm on all the rows of a matrix

This program contains three stages:

In the first stage:

- $\quad$ All the PE's are set in Simple Bridge of direction WE.

- $\quad$ All the PE's of rows 0, 2, 3 and 4 are marked.

Each marked PE computes a random value and stores it in its register $\mathrm{reg}[0]$.
Finding the representative PE of each row using a macro command $<$ defineRepresentativeForEachRow $/>$.

The second stage is devoted to a «while» loop, where:

1) The marco command «doDistributeParityIndex from $=$ 'W' $\gg$ is used to label the marked PE's alternatively by 0 or 1 . 
2) In each row, all the PE's of even labels send their data on their West ports. These data will be received on East ports of the corresponding marked PE's having odd labels. Each PE stores the received data in its register $\operatorname{reg}[1]$.

3) Each PE having even label and having sent its data, becomes unmarked and turns back to the stand by mode before setting itself in (SB-WE) state ( this PE is died).

4) Each PE having received data on its East ports computes the minimum value among its reg [0] and reg[1] contents and stores the result in reg [0].

5) The operations from 1) to 4) constitute the instructions of a loop that is repeated while there exist in any row of the matrix the PE's that are not in stand by mode. This loop is finished when it remains in life just the representative $\mathrm{PE}$ at each row.

The remained instruction of this stage corresponds to the back up operations that are used to distribute at each row the minimum value obtained by its representative PE after the last loop.

The third stage is used to mark all the PE's having the minimum value in their rows. It is based on the following instruction:

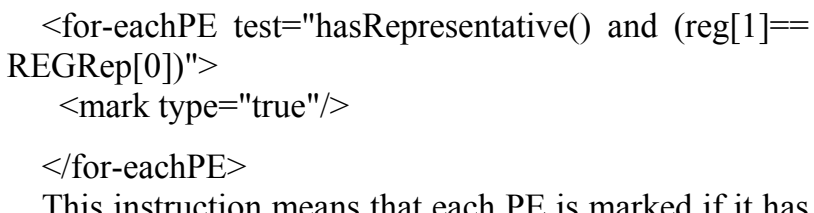
representative $\mathrm{PE}$ and its own value in its register reg [1] equals the value of register $\mathrm{reg}[0]$ of its representative PE.

This stage allows us to display at each row, the PE having the minimum value. See Figure 11 (d).

Remark: When two or more PE's have the same minimal value on the same row, they are displayed together and they declare themselves having the minimal value.

\section{Conclusions}

In this paper, we have presented an important tool for the parallel programmers to validate their parallel computing algorithms. The developped virtual machine emulates a SIMD structure re-configurable mesh computer. The obtained parallel virtual machine and its programming language compiler can be used as a high performance computing system. It represents in our laboratory a very important tool to validate our algorithms in the parallel image processing domain.

Actually, the cost of physical parallel architectures remains very high. Subsequently, their use is very limited over the world. In this context, we start this first part of this project and we continue by developing further related works. They are oriented to model other topological parallel structures, such as: pyramid and hypercube architectures. All the components of this work are subscribed in a strategy of popularizing the parallel computing domain.

\section{REFERENCES}

[1] R. Miller. et al, "Geometric algorithms for digitized pictures on a mesh connected computer," IEEE Transactions on PAMI, Vol. 7, No. 2, pp. 216-228, 1985.

[2] V. K. Prasanna and D. I. Reisis, "Image computation on meshes with multiple broadcast," IEEE Transactions on PAMI, Vol. 11, No. 11, pp. 1194-1201, 1989.

[3] H. LI, M. Maresca, "Polymorphic torus network," IEEE Transaction on Computer, Vol. C-38, No. 9, pp. 1345$1351,1989$.

[4] T. Hayachi, K. Nakano, and S. Olariu, "An O $\left((\log \log n)^{2}\right)$ time algorithm to compute the convex hull of sorted points on re-configurable meshes," IEEE Transactions on Parallel and Distributed Systems, Vol. 9, No. 12, 11671179, 1998.

[5] R. Miller, V. K. Prasanna-Kummar, D. I. Reisis, and Q. F. Stout, "Parallel computation on re-configurable meshes," IEEE Transactions on Computer, Vol. 42, No. 6, pp. 678-692, 1993.

[6] J. P Gray and T. A. Kean, "Configurable hardware: A new paradigm for computation," Proceedings of $10^{\text {th }} \mathrm{Cal}$. Tech. conference on VLSI, pp. 279-295, 1989.

[7] H. Li and M. Maresca, "Polymorphic torus architecture for computer vision," IEEE Transactions on PAMI, Vol. 11, No. 3, pp. 233-242, 1989.

[8] D. B. Shu, G. Nash, and C. Weems, "Image understanding architecture and applications, in advances in machine vision, J. L. C. SANZ, Ed. Springer-Verlag, NY, pp. 297 355,1989 .

[9] H. M. Alnuweiri et al., "Re-configurable network switchmodels," Technical Report, CICSR, Univ. British Columbia, 1993.

[10] J. L. Trahan, R. K. Thiruchelvan, and R. Vaidyanathan, "On the power of segmenting and fusing buses, Proc. Int. Parallel Processing Symposium, pp. 79-83, 1993.

[11] R. Miller and Q. F. Stout, "Mesh computer algorithms for computational geometry," IEEE Transactions on computer, Vol. 38, No. 3, pp. 321-340, 1989.

[12] J. Elmesbahi, O. Bouattane, and Z. Benabbou, "O(1) time quadtree algorithm and its application for image geometric properties on a Mesh Connected Computer (MCC), IEEE Trans. On Systems, Man, and Cybernetics, Vol. 25, No. 12, pp. 1640-1648, 1995.

[13] J. Elmesbahi, O. Bouattane, A. Raihani, M. Elkhaili, A. Rabbaa, "Parallel algorithms representation of points and curvilinear data," International Journal of SAMS, Vol. 33, pp. 479-494, 1998.

[14] O. Bouattane, J. Elmesbahi, M. Khaldoun, and A. Rami, "A fast algorithm for k-nearest neighbor problem on a reconfigurable mesh computer," Journal of Intelligent and Robotic Systems, Kluwer academic Publisher, Vol. 32, pp. 
347-360, 2001

[15] R. Miller et al, "Meshes with re-configurable Buses," Proceedings of $5^{\text {th }}$ MIT Conference on Advanced Research in VLSI, Cambridge, MA, pp. 163-178, 1988.

[16] Ling Tony et al, Efficient parallel processing of image contours," IEEE Transactions on PAMI, Vol. 15, No. 1, pp. 69-81, 1993.

[17] O. Bouattane, J. Elmesbahi, and A. Rami, “A fast parallel algorithm for convex hull problem of multi-leveled images," Journal of Intelligent and Robotic Systems, Kluwer academic Publisher, Vol. 33, pp. 285-299, 2002.

[18] A. Errami, M. Khaldoun, J. Elmesbahi, and O. Bouattane, "O(1) time algorithm for structural characterization of multi-leveled images and its applications on a re-configurable mesh computer," Journal of Intelligent and Robotic Systems, Springer, Vol. 44, pp. 277-290, 2006.

[19] M. Migliardi and V. Sunderam, "Emulating parallel programming environments in the harness meta computing system," Parallel Processing Letters, Vol. 11, No. 2 \& 3, pp. 281-295, 2001.
[20] D. Kurzyniec, V. Sunderam, and M. Migliardi, "PVM emulation in the harness metacomputing framework -design and performance evaluation," Proc. of the Second IEEE International Symposium on Cluster Computing and the Grid (CCGrid), Berlin, Germany, pp. 282-283, May 2002.

[21] M. Migliardi, P. Baglietto, and M. Maresca, "Virtual parallelism allows relaxing the synchronization constraints of SIMD computing paradigm, Proceedings of HPCN98, Amsterdam (NL), pp. 784-793, April 1998.

[22] M. Migliardi and M. Maresca, "Modeling instruction level parallel architectures efficiency in image processing applications," Proceedings of of HPCN97, Lecture Notes in Computer Science, Springer Verlag, Vol. 1225, pp. 738-751, 1997.

[23] M. Migliardi and R. Podesta, "Parallel component descriptor language: XML based deployment descriptors for grid application components," Proc. of the 2007 International Conference on Parallel and Distributed Processing Techniques and Applications, Las Vegas, Nevada, USA, pp. 955-961, June 2007. 